\title{
Progressive Parametric Query Optimization
}

\author{
Pedro Bizarro, Nicolas Bruno, and David J. DeWitt
}

\begin{abstract}
Commercial applications usually rely on precompiled parameterized procedures to interact with a database. Unfortunately, executing a procedure with a set of parameters different from those used at compilation time may be arbitrarily suboptimal. Parametric query optimization (PQO) attempts to solve this problem by exhaustively determining the optimal plans at each point of the parameter space at compile time. However, PQO is likely not cost-effective if the query is executed infrequently or if it is executed with values only within a subset of the parameter space. In this paper, we propose instead to progressively explore the parameter space and build a parametric plan during several executions of the same query. We introduce algorithms that, as parametric plans are populated, are able to frequently bypass the optimizer but still execute optimal or near-optimal plans.
\end{abstract}

Index Terms-Parametric query optimization, adaptive optimization, selectivity estimation.

\section{INTRODUCTION}

I $\mathrm{N}$ many applications, the values of runtime parameters of 1 the system, data, or queries themselves are unknown when queries are originally optimized. In these scenarios, there are typically two trivial alternatives to deal with the optimization and execution of such parameterized queries. One approach, termed here as Optimize-Always, is to call the optimizer and generate a new execution plan every time a new instance of the query is invoked. Another trivial approach, termed Optimize-Once, is to optimize the query just once, with some set of parameter values, and reuse the resulting physical plan for any subsequent set of parameters. Both approaches have clear disadvantages. OptimizeAlways requires an optimization call for each execution of a query instance. These optimization calls may be a significant part of the total query execution time, especially for simple queries. In addition, Optimize-Always may limit the number of concurrent queries in the system, as the optimization process itself may consume too much memory. On the other hand, Optimize-Once returns a single plan that is used for all points in the parameter space. The chosen plan may be arbitrarily suboptimal for parameter values different from those for which the query was originally optimized.

\subsection{Parametric Query Optimization}

An alternative to Optimize-Always and Optimize-Once is Parametric Query Optimization (PQO). At optimization time, PQO determines a set of plans such that for each point in the parameter space, there is at least one plan in the set that it is optimal. The regions of optimality of each plan are also computed. Later, when an instance of the query is submitted, PQO chooses the best precomputed plan for the query

- P. Bizarro is with the CISUC/DEI, University of Coimbra, DEI-Polo 2, 3030-290 Coimbra, Portugal. E-mail: bizarro@dei.uc.pt.

- N. Bruno is with Microsoft Research, One Microsoft Way, Redmond, WA 98052. E-mail: nicolasb@microsoft.com.

- D.J. DeWitt is with the University of Wisconsin, Madison, 1210 W. Dayton Street, Madison, WI 53706. E-mail: dewitt@cs.wisc.edu.

Manuscript received 13 Nov. 2007; revised 9 May 2008; accepted 17 July 2008; published online 25 July 2008.

Recommended for acceptance by $S$. Wang.

For information on obtaining reprints of this article, please send e-mail to: tkde@computer.org, and reference IEEECS Log Number TKDE-2007-11-0559. Digital Object Identifier no. 10.1109/TKDE.2008.160. instance and executes it without making a new optimization call. PQO proposals often assume that the cost formulas of physical plans are linear or piecewise linear with respect to the cost parameters and that the regions of optimality are connected and convex. However, in reality, the cost functions of physical plans and regions of optimality are not so well behaved. A more important problem results from the fact that PQO has a much higher start-up cost than optimizing a query a single time (PQO usually requires several invocations of the optimizer with different parameters [8], [9]). When a previously unseen query arrives, it is therefore not clear to determine whether PQO should be used: it may not be cost-effective to solve the full PQO problem if the query is not executed frequently or if it is repeatedly executed with values covering a small subspace of the entire parameter space. Most previous work (see Section 6) ignores this dilemma and instead solves the full PQO problem, potentially wasting more resources than necessary.

\subsection{Contributions}

In this paper, we propose an alternative approach to handle parametric queries that addresses the shortcomings described above. Our contributions are listed as follows:

- In Section 2, we propose Progressive Parametric Query Optimization (PPQO), a novel framework to improve the performance of processing parameterized queries. We also propose the Parametric Plan (PP) interface as a way to incorporate PPQO in DBMS.

- In Sections 3 and 4, we propose two implementations of PPQO with different goals. On one hand, Bounded has proven optimality guarantees. On the other hand, Ellipse results in higher hit rates and better scalability.

- Finally, in Section 5, we present an extensive performance evaluation of PPQO using a prototype implementation on Microsoft SQL Server 2005.

\section{Progressive Parametric Query OPTIMIZATION}

The main idea of PPQO is to incrementally solve (or approximate) the solution to the PQO problem as successive 


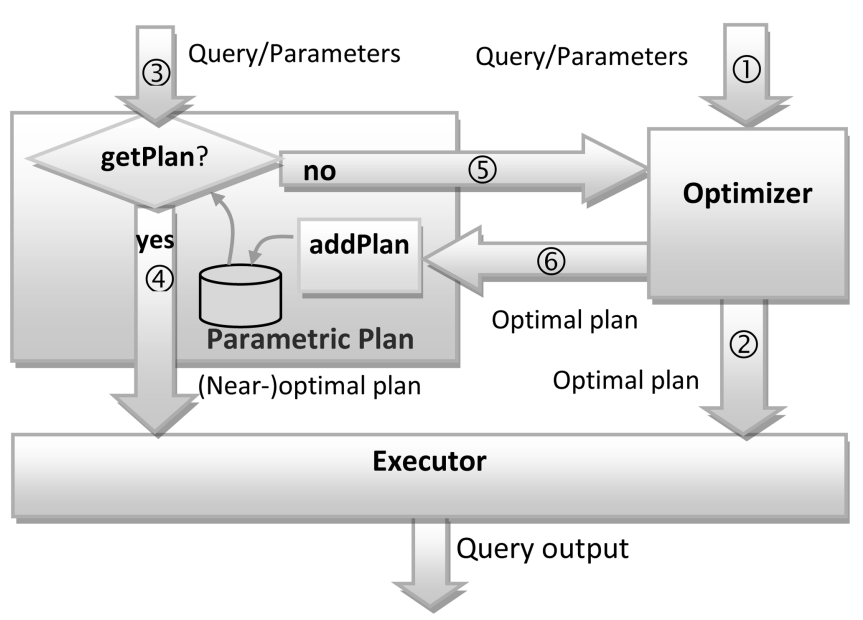

Fig. 1. Using PPs to process a query.

query execution calls are submitted to the DBMS. Fig. 1 shows a high-level architecture of our approach. Given a query and its parameter values, a traditional optimizer returns the optimal execution plan along with its estimated cost (1) and (2) in the figure). In contrast, a PPQO-enabled optimizer introduces a data structure called PP, which incrementally maintains plans and optimality regions, allowing us to reuse work across optimizations. As the PP data structure becomes populated, it is possible to completely bypass the optimization process without hurting the quality of the resulting execution plans.

When a new instance of a parametric query arrives (3) in Fig. 1), PPQO tries to obtain an optimal (or near-optimal) plan by consulting the PP data structure. If it is successful, it returns such plan, and a full optimization call is avoided (4) in Fig. 1). Otherwise, it makes an optimization call (5) in Fig. 1), and both the resulting optimal plan and cost are added to the PP for future use (6) in Fig. 1). Due to the size of the parameter space, PPs should not be implemented as exact lookup caches of plans because there would be too many "cache misses." Also, due to the nonlinear and discontinuous nature of cost functions, PPs should not be implemented as nearest neighbor lookup structures as there will be no guarantee that the optimal plan of the nearest neighbor is optimal or close to optimal for the point in the parameter space being considered [3], [16]. We now describe the PPQO problem in more detail, borrowing notation and definitions from the classic parametric optimization problem.

\subsection{Definitions and Preliminaries}

A parametric query $Q$ is a text representation of a relational query with placeholders for $m$ parameters $v p t=$ $\left(v_{1}, \ldots, v_{m}\right)$. Vector $v p t$ is called a ValuePoint. Examples of parameter values are system parameters (e.g., available memory) and query-dependant parameters (e.g., constants in parametric predicates). In the rest of the paper, we focus on query-dependant parameters since they cover the most common scenarios. We note, however, that our techniques can also be adapted to other kinds of parameters.

Using vpt directly to model the parameter space and characterize regions of optimality for plans is in general difficult (see below for an example). To address this problem, we use a transformation function $\varphi$, which is optimizer specific and transforms ValuePoints into what we call CostPoints. A CostPoint is a vector $c p t=\left(c_{1}, \ldots, c_{n}\right)$, where each $c_{i}$ is a cost parameter with an ordered domain. A wellknown implementation of $\varphi$, which we justify below and use in the rest of the paper, is transforming parametric predicate values into the corresponding predicate selectivities. For instance, consider predicate age $<\$ \mathrm{X} \$$, with parameter $\$ \mathrm{X} \$$. Function $\varphi$ would then map a specific constant $\mathrm{c}$ for $\$ \mathrm{X} \$$ into the selectivity of the nonparametric predicate age $<\mathrm{c}$.

Let $p$ be some execution plan that evaluates query $Q$ for a given vpt. The cost function of $p$, denoted $p(c p t)$, takes a CostPoint cpt as an input and returns the cost of evaluating plan $p$ under $c p t$. For every legal value of the parameters, there is some plan that is optimal. Given a parametric query $Q$, the maximum parametric set of plans (MPSP) is the set of plans, each of which is optimal for some point in the $n$-dimensional cost-based parameter space. The region of optimality for plan $p$, denoted $r(p)$, is defined as

$$
r(p)=\left\{\left(t_{1}, \ldots, t_{n}\right) \mid p \text { is optimal at }\left(c_{1}=t_{1}, \ldots, c_{n}=t_{n}\right)\right\} .
$$

Finally, a parametric optimal set of plans (POSP) is a minimal subset of MPSP that includes at least one optimal plan for each point in the parameter space.

Having introduced this basic terminology, we next justify the need for the transformation function $\varphi$ and then define the PPQO framework in detail.

\subsection{The Parameter Transformation Function $\varphi$}

Recall that a value parameter refers to an input value of the parametric SQL query to execute. On the other hand, a cost parameter is an input parameter in the formulas used by the optimizer to estimate the cost of a query plan. Cost parameters are estimated during query optimization from value parameters and from information in the database catalog. (Physical characteristics that affect the cost of plans but do not depend on query parameters, such as the average tuple size or the cost of a random $\mathrm{I} / \mathrm{O}$, are considered physical constants instead of cost parameters.)

A crucial cost parameter that is used during optimization is the estimated number of tuples in (intermediate) relations processed by the query plan: most query plans have cost formulas that are monotonic in the number of tuples processed by the query. On the other hand, there is no obvious relationship between the value parameters and the cost of the query plans. Thus, it becomes much easier to characterize the regions of optimality using a cost-based parameter space than using a value-based parameter space. In Example 1 below and in what follows, we use a costbased parameter space whose dimensions are predicate selectivities. (Note that the estimated number of tuples of each relation processed by a query is typically derived from selectivities of subexpressions computed during query optimization.)

Example 1. Table FRESHMEN(NAME, AGE) succinctly describes first-year graduate students. The age distribution of students is showed in Fig. 2. Consider queries of the following form:

SELECT *

FROM FRESHMEN

WHERE AGE $=\$ X \$$ OR AGE $=\$ Y \$$ 


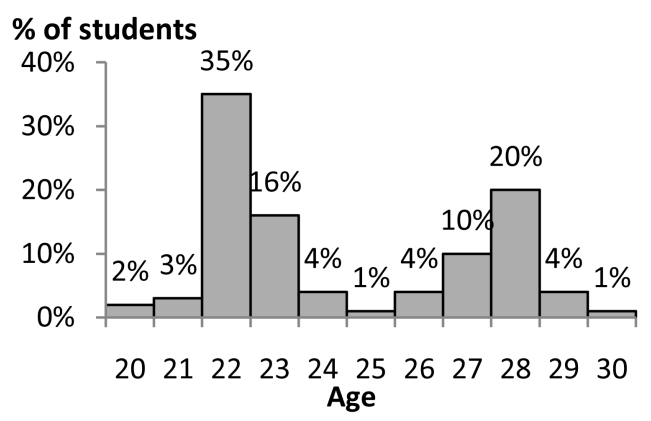

Fig. 2. Age distribution in table FRESHMEN.

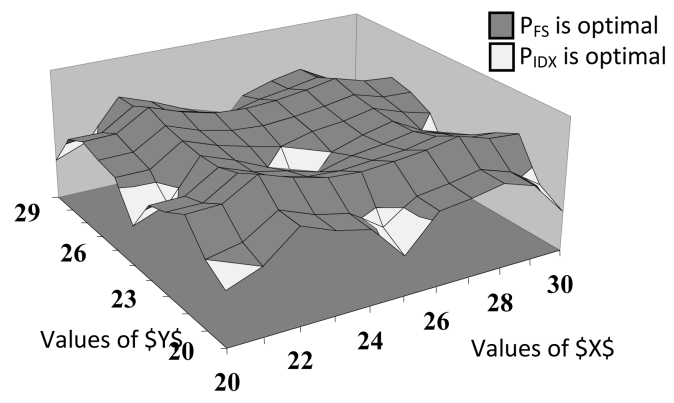

Fig. 3. Value-based parameter space.

Assume that the optimal plan for queries that retrieve less than 5 percent of FRESHMEN tuples is $\mathrm{P}_{\mathrm{IDX}}$, a plan using an index on column AGE. For all other queries, the optimal plan is $\mathrm{P}_{\mathrm{FS}}$, a full-table scan on FRESHMEN. The parameters of this query can be represented as the absolute values used for parameters $\$ \mathrm{X} \$$ and $\$ \mathrm{Y} \$$ or as the selectivities of predicate age $=\$ \mathrm{X} \$$ and predicate age $=\$ Y \$$. Accordingly, the costs of physical $P_{I D X}$ and $\mathrm{P}_{\mathrm{FS}}$ can be represented in value-based parameter spaces, shown in Fig. 3, or in selectivity-based (also referred to as cost-based) parameter spaces, shown in Fig. 4. Clearly, the selectivity-based representation results in a much more manageable parameter space than the (seemingly chaotic) value-based representation. The reason is that selectivity-based representations are better aligned to the optimizer cost model and tend to be represented by monotonic cost functions, and therefore, the regions of optimality of plans tend to cluster together.

In the rest of this paper, we assume that function $\varphi$ takes query $Q$ and its SQL parameters, $v p t$, and returns $c p t$ as a vector of selectivities. Computing the selectivities in $c p t$ corresponds to the task of selectivity estimation, a subroutine inside of query optimization. Other components of query optimization-e.g., plan enumeration, rule transformation, and costing-need not be part of the implementation of function $\varphi$. In general, computing selectivity values from actual values is done by manipulating in-memory histograms, which is very efficient, and a negligible fraction of the full query optimization task.

We note that the arity of the value-based parameter space and that of the selectivity-based parameter space are not necessarily the same. On one hand, it is possible to have predicates of the form age $>\$ \mathrm{X} \$$ and age $<\$ \mathrm{Y} \$$, where two value predicates are collapsed into a single selectivity value for the combined predicate. Similarly, a query that contains

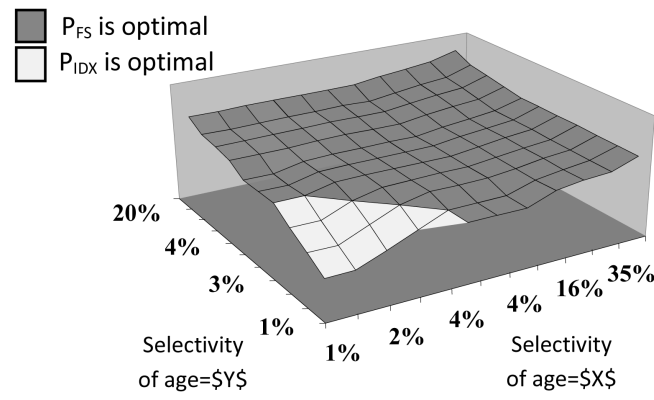

Fig. 4. Selectivity-based parameter space.

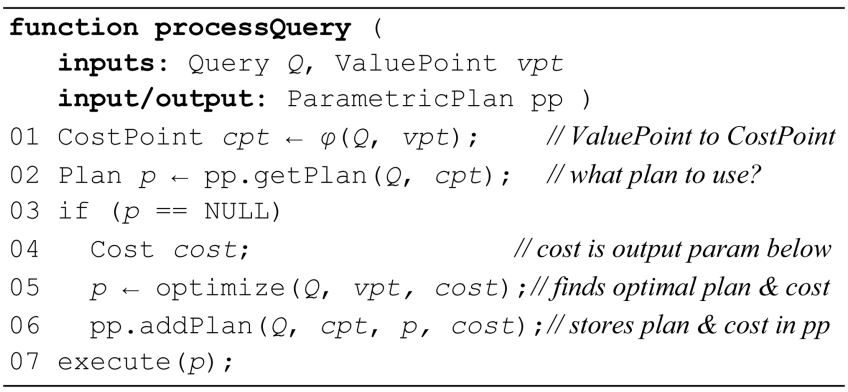

Fig. 5. Using PPs.

a predicate of the form R.age $<\$ \mathrm{X} \$$ and also a join between tables $\mathrm{R}$ and $\mathrm{S}$ might require two selectivity parameters to capture the optimizer's cost model: one for the selectivity of the predicate on the base table and another for the selectivity of the predicate on the join. In our prototype and experimental evaluation, we use a simple one-to-one mapping between parametric predicates and selectivity values (i.e., we do not consider join predicates nor combine atomic predicates over the same column). The reasons behind our choice are the following: 1) this is the mapping used in previous work on parametric optimization, 2) it can be implemented without deep knowledge about the underlying query optimizer, and 3) our experiments show that this simple model is very competitive.

\subsection{The Parametric Plan Interface}

We now give an operational description of the PP component of PPQO by describing its two main operations (also see Fig. 1):

- $\operatorname{addPlan}(Q, c p t, p, c)$. This operation registers that plan $p$, with estimated $\operatorname{cost} c$, is optimal for query $Q$ at CostPoint cpt.

- $\operatorname{getPlan}(Q, c p t)$. This operation returns the plan that should be used for query $Q$ and cost values cpt or returns null if no plan is considered good enough for $Q$.

Implementations of the PP interface are used during query processing, as shown in Fig. 1 and in the pseudocode in Fig. 5. When parametric query parameter instances are required to execute, the DBMS calls the PP's getPlan method. If getPlan returns plan $p_{1}$, then $p_{1}$ is used for execution, and an optimization call is avoided. If getPlan returns null (we call this situation a getPlan miss), then the optimizer is called, and a potentially new plan, $p_{2}$, is obtained from the optimizer. Plan $p_{2}$ is then executed. The parameter 


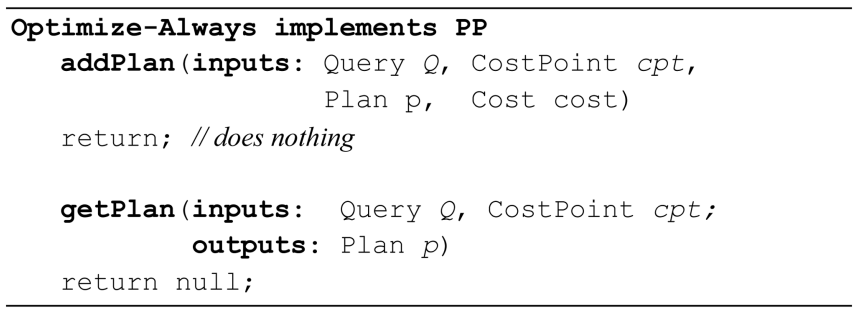

Fig. 6. Optimize-Always implementation.

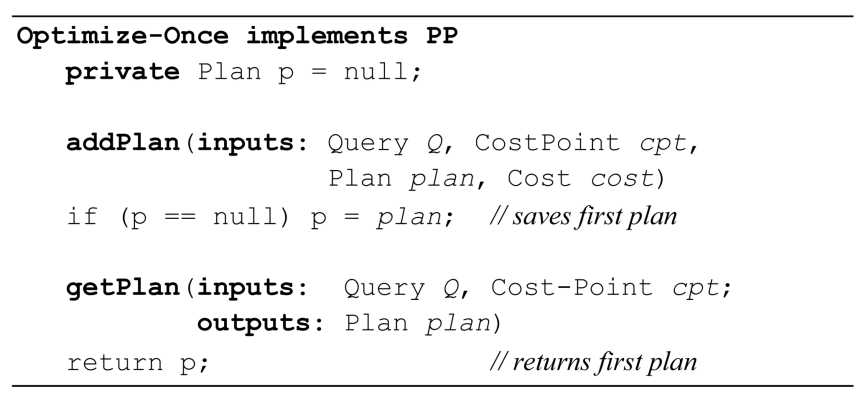

Fig. 7. Optimize-Once implementation.

values, plan $p_{2}$, and its cost are then added to the PP using addPlan.

As we show in Sections 3 and 4, the PP interface can be used to implement various PPQO policies. However, it can also implement simple policies like Optimize-Always and Optimize-Once. Fig. 6 shows the Optimize-Always implementation of the PP interface, in which addPlan is empty and getPlan always returns null, forcing an optimization for every query. Fig. 7 shows the Optimize-Once implementation of the PP interface, in which addPlan saves the first plan it is given as input and getPlan returns such plan in all subsequent calls.

\subsection{Parametrics Plans: Requirements and Goals}

The main trade-off in PPQO is to avoid as many optimization calls as possible as long as we are willing to execute suboptimal-but close to optimal—plans (note that this goal has also been proposed in [5] and [11] in the context of classical PQO). Thus, PP implementations must obey the Inference Requirement below.

Inference Requirement. After a number of addPlan calls, there must be cases where getPlan returns an (near-)optimal plan $p$ for query $Q$ and parameter point $c p t$, even if $\operatorname{add} \operatorname{Plan}(Q, c p t, p, \operatorname{cost})$ was never called.

Given a sequence of execution requests of the same query with potentially different input parameters, PPQO has therefore two conflicting goals:

- Goal 1. Minimize the number of optimization calls.

- Goal 2. Execute plans with costs as close to the cost of the optimal plan as possible.

Consider a trivial cache implementation of the PP interface, which stores $(Q, c p t)$ pairs as the lookup key and $(p, c o s t)$ as the inserted value. This implementation cannot fulfill the inference requirement because it would return hits only for previously inserted $(Q, c p t)$ pairs. In the next sections, we propose two PPQO implementations, each giving priority to one of the above goals. Bounded-PPQO,

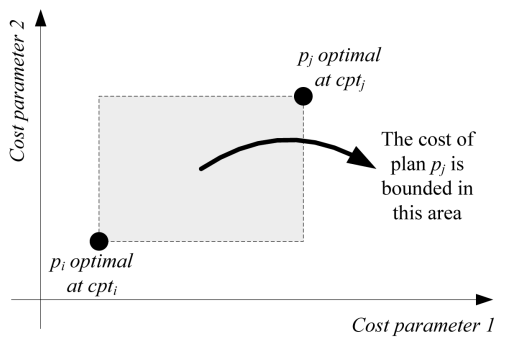

Fig. 8. Overview of Bounded-PPQO.

described in Section 3, gives priority to Goal 2. Ellipse$P P Q O$, described in Section 4, gives priority to Goal 1.

\section{THE BOUNDED-PPQO IMPLEMENTATION}

We now describe the first of two proposed PPQO implementations, termed Bounded-PPQO or simply Bounded. This implementation provides guarantees on the quality of the plans returned by $\operatorname{get} \operatorname{Plan}(Q, c p t)$, thus focusing on Goal 2 of PPQO (see previous section). Either the returned plan $p$ is null (and an optimization call cannot be avoided) or $p$ has a cost guaranteed to be within a userspecified bound of the cost of the optimal plan. Specifically, the cost of plan $p$ returned by getNext is guaranteed to be bounded by $O p t \operatorname{Cost}^{*} M+A$, where OptCost is the cost of the optimal plan, and $M \geq 1$ and $A \geq 0$ are user-defined constants. Both $M$ and $A$ can be used to specify different bounds on suboptimality and are generally application specific. (We report, however, the effects of varying parameters $M$ and $A$ in Section 5.)

The intuition for the Bounded-PPQO implementation is given as follows: Consider a parametric query with two parameters. If plans $p_{i}$ and $p_{j}$ are optimal in some CostPoints $c_{i} t_{i}$ and $c p t_{j}$, which delimit a box as shown in the twodimensional example in Fig. 8, then we can provably bound the cost of plan $p_{j}$ in all points within that box if the cost functions are monotonic along all dimensions (e.g., if the cost of the query increases whenever the selectivity of any parameter increases). Specifically, the cost of plan $p_{j}$ in the box will be between the cost of plan $p_{i}$ at $c p t_{i}$ and the cost of plan $p_{j}$ at $c p t_{j}$.

\subsection{Preliminaries}

We now introduce some definitions required to describe the Bounded-PPQO implementation:

- Relationship equal ( $\equiv)$. Given $c p t_{1}=\left(c_{1,1}, \ldots, c_{1, n}\right)$ and $c p t_{2}=\left(c_{2,1}, \ldots, c_{2, n}\right), c p t_{1} \equiv c p t_{2}$ iff $\forall i c_{1, i}=c_{2, i}$.

- Relationships below (४) and above (\$). Given $c p t_{1}=$ $\left(c_{1,1}, \ldots, c_{1, n}\right)$ and $\quad \operatorname{cpt}_{2}=\left(c_{2,1}, \ldots, c_{2, n}\right), \quad \operatorname{cpt}_{1} \varangle c t_{2}$, $\left(c p t_{1}>c p t_{2}\right)$ iff $\forall i \quad c_{1, i} \leq c_{2, i}\left(c_{1, i} \geq c_{2, i}\right)$, and $\exists i$, $c_{1, i} \neq c_{2, i}$. Note that both $\varangle$ and $>$ are transitive. That is, if $c p t_{1} \triangleleft c p t_{2}\left(c p t_{1} \gg c p t_{2}\right)$ and $c p t_{2} \varangle c p t_{3}\left(c p t_{2} \gg c p t_{3}\right)$, then $\mathrm{cpt}_{1} \triangleleft \mathrm{cpt}_{3}\left(\mathrm{cpt} t_{1}>\mathrm{cpt} \mathrm{t}_{3}\right)$.

- Opt(cpt). It is the cost of an optimal plan at cpt.

- Bounding pair. Triples $t_{i}=\left(c t_{i}\right.$, plan $\left._{i}, \operatorname{cost}_{i}\right)$ and $t_{j}=\left(c p t_{j}\right.$, plan $_{j}$, cost $\left._{j}\right)$ are a bounding pair if plan $\operatorname{plan}_{i}\left(\operatorname{plan}_{j}\right)$ is an optimal plan at $\operatorname{cpt}_{i}\left(\mathrm{cpt}_{j}\right)$ with cost $\operatorname{cost}_{i}\left(\operatorname{cost}_{j}\right), \operatorname{cpt}_{i} \triangleleft \operatorname{cpt}_{j}$, and 


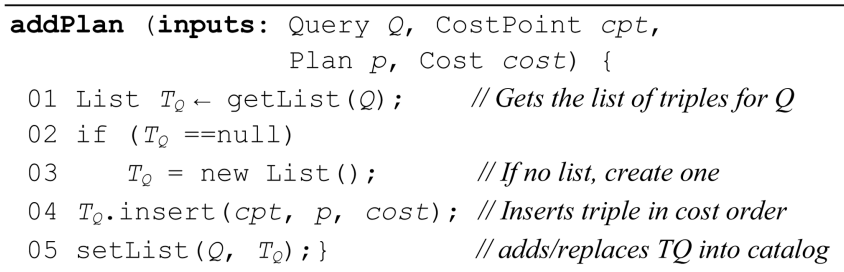

Fig. 9. Bounded's addPlan implementation.

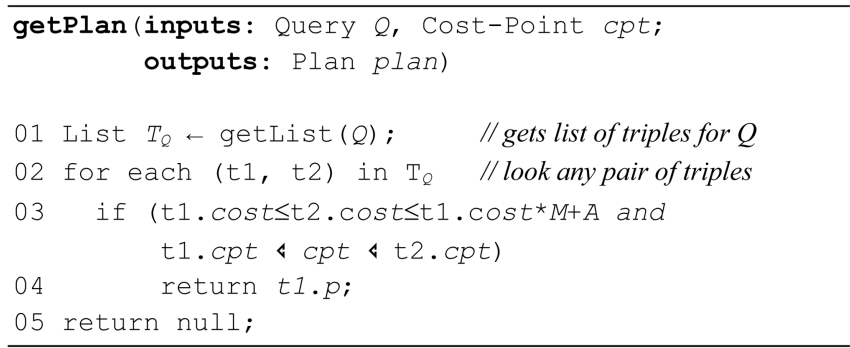

Fig. 10. Bounded's getPlan implementation.

$$
\operatorname{plan}_{i}\left(c p t_{i}\right) \leq \operatorname{plan}_{j}\left(c p t_{j}\right) \leq \operatorname{plan}_{i}\left(c p t_{i}\right)^{*} M+A,
$$

where $M$ and $A$ are, respectively, any user-defined multiplicative and additive factors, with $M \geq 1$ and $A \geq 0$. The pair $\left(t_{i}, t_{j}\right)$ is also said to bound cpt if cpt $_{i} \triangleleft c p t \varangle c p t_{j}$.

We additionally rely on the intuitive Monotonic Assumption (or $M A$ ), stated as follows: given plan $p$ and CostPoints $c p t_{1}$ and $c p t_{2}$, if $c p t_{1} \triangleleft c p t_{2}$, then $p\left(c p t_{1}\right) \leq p\left(c p t_{2}\right){ }^{1}$

\subsection{Implementation of addPlan for Bounded}

Function addPlan $(Q, c p t, p, c o s t)$, shown in Fig. 9, associates with each parametric query $Q$ a list $T_{Q}$ of triples $(c p t, p, c o s t)$ ordered by cost, where $p$ is an optimal plan at $c p t$ with an estimated execution cost (at $c p t$ ) of $\operatorname{cost}=p(c p t)$.

\subsection{Implementation of getPlan for Bounded}

For user-defined constants $M \geq 1$ and $A \geq 0$, Bounded's getPlan $(Q, c p t)$ searches for a pair $t_{i}=\left(c p t_{i}\right.$, plan $_{i}$, cost $\left._{i}\right)$ and $t_{j}=\left(c p t_{j}\right.$, plan $\left._{j}, \operatorname{cost}_{j}\right)$ that bounds cpt (i.e., with cost $_{i} \leq$ cost $_{j} \leq \operatorname{cost}_{i}{ }^{*} M+A$ and with $\left.c p t_{i} \triangleleft c p t \varangle c p t_{j}\right)$. If it finds no such bounding pair, getPlan returns null. Otherwise, it returns such plan (see Fig. 10 for a high-level description).

We next show that if getPlan returns plan $p$, it guarantees under the MA that the cost of executing $p$ at $c p t$ satisfies $O p t(c p t) \leq p(c p t) \leq O p t(c p t)^{*} M+A$. We first show in Lemma 1 that if the MA holds for every plan considered, then the cost of the optimal plan at any point (regardless of what the optimal plan is at any single point) also increases monotonically with the parameters.

Lemma 1. If $c p t_{1} \varangle c p t_{2}, \operatorname{cost}_{1}=p_{1}\left(c p t_{1}\right)=O p t\left(c p t_{1}\right)$, and cost $_{2}=p_{2}\left(c p t_{2}\right)=O p t\left(c p t_{2}\right)$, then cost $_{1} \leq \operatorname{cost}_{2}$.

1. All cost parameters we use are selectivities. Since higher selectivities imply more tuples to process, the MA follows the intuition that plans that process more tuples likely cost more than plans that process less tuples. Although not true for all queries-e.g., queries using SQL clause NOT EXISTS may have nonmonotonic costs-plans with nonmonotonic costs are less common than plans with costs monotonic with the number of processed tuples.

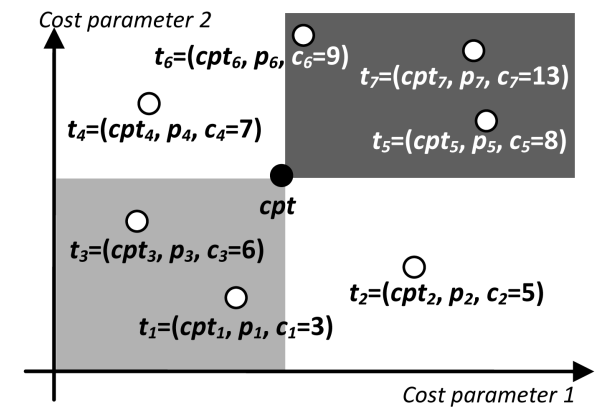

Fig. 11. $T_{Q}=(t 1, t 2, t 3, t 4, t 5, t 6, t 7)$.

Proof. We note that if $p_{2}$ is optimal at $c p t_{1}$, then cost $_{1}=p_{2}\left(c p t_{1}\right)$. Otherwise, $p_{2}$ is not optimal at $c p t_{1}$, and therefore, $\operatorname{cost}_{1}<p_{2}\left(c p t_{1}\right)$. In any case, we have that cost $_{1} \leq p_{2}\left(c p t_{1}\right)$, which, coupled with the MA and $c p_{t 1} \triangleleft c p_{t 2}$, implies that $p_{2}\left(c p_{t 1}\right) \leq p_{2}\left(c p_{t 2}\right)=c o s t_{2}$. Putting the last two inequalities together, we obtain $\operatorname{cost}_{1} \leq \operatorname{cost}_{2}$.

Lemma 2. If $M \geq 1$, $\operatorname{cost}_{x} \leq \operatorname{cost}_{z} \leq \operatorname{cost}_{x} * M+A$, and $\operatorname{cost}_{x} \leq \operatorname{cost}_{y} \leq \operatorname{cost}_{z}$, then $\operatorname{cost}_{y} \leq \operatorname{cost}_{z} \leq \operatorname{cost}_{y}{ }^{*} M+A$.

Proof. Since $M \geq 1$ and $\operatorname{cost}_{x} \leq$ cost $_{y}$, it follows that $\operatorname{cost}_{x}{ }^{*} M+A \leq \operatorname{cost}_{y}{ }^{*} M+A$. Also, since $\operatorname{cost}_{x} \leq \operatorname{cost}_{z} \leq$ $\operatorname{cost}_{x}{ }^{*} M+A$, it follows that $\operatorname{cost}_{z} \leq \operatorname{cost}_{x}{ }^{*} M+A \leq$ cost $_{y} * M+A$. Finally, since cost $_{x} \leq$ cost $_{y} \leq$ cost $_{z}$, it follows that cost $_{y} \leq \operatorname{cost}_{z} \leq \operatorname{cost}_{y}{ }^{*} M+A$.

Finally, Theorem 1 establishes our desired result.

Th e orem 1. If $t_{i}=\left(c p t_{i}\right.$, plan $_{i}$, cost $\left._{i}\right)$ and $t_{j}=$ $\left(c p t_{j}\right.$, plan $_{j}$, cost $\left._{j}\right)$ are a bounding pair for some $M \geq 1$ and $A \geq 0$, then under the $M A$, the cost of plan can $_{\text {be tightly }}$ bounded such that $O p t(c p t) \leq p l a n_{j}(c p t) \leq O p t(c p t){ }^{*} M+A$ for all cpt such that cpt $t_{i}<p t \triangleleft c p t_{j}$.

Proof. By Lemma 1 and $c p t_{i} \varangle c p t \varangle c p t_{j}$, it follows that cost $t_{i} \leq$ $O p t(c p t) \leq \operatorname{cost}_{j}$. Also, by Lemma 2 and cost $_{i} \leq \operatorname{cost}_{j} \leq$ cost $_{i}{ }^{*} M+A$, we get $O p t(c p t) \leq \operatorname{cost}_{j} \leq O p t(c p t){ }^{*} M+A$.

Example 2. For some query $Q$, assume that addPlan was already called for the points (and associated triples) shown in Fig. 11 (i.e., assume that the PP stores information about the optimal plans and costs for the triples in $T_{Q}=\left(t_{1}, t_{2}, t_{3}, t_{4}, t_{5}, t_{6}, t_{7}\right)$ ). Given cpt (shown as a black circle) in the cost-based parameter space, $M=1.5$, and $A=0$, which plan would getPlan $(Q, c p t)$ return? There are six pairs $\left(c p t_{i}, c p t_{j}\right)$ such that $c p t_{i} \varangle c p t \varangle c p t_{j}:\left(c p t_{1}, c p t_{6}\right)$, $\left(c p t_{1}, c p t_{7}\right),\left(c p t_{3}, c p t_{5}\right),\left(c p t_{3}, c p t_{6}\right)$, and $\left(c p t_{3}, c p t_{7}\right)$. From those pairs, only two triples bound $c p t$ : pair $\left(t_{3}, t_{5}\right)$, because $c_{3} \leq c_{5} \leq c_{3}{ }^{*} 1.5+0 \Longleftrightarrow 6 \leq 8 \leq 9$, and pair $\left(t_{3}, t_{6}\right)$, because $c_{3} \leq c_{6} \leq c_{3}{ }^{*} 1.5+0 \Longleftrightarrow 6 \leq 9 \leq 9$. Thus, either plan $p_{5}$ and plan $p_{6}$ can be safely returned by getPlan.

\subsection{Efficient Implementation of getPlan}

The naïve implementation of getPlan in Fig. 10 enumerates all pairs of tuples $\left(t_{i}, t_{j}\right) \in T_{Q} \times T_{Q}, t_{i} \neq t_{j}$, that were introduced by addPlan and tests if any pair bounds cpt. If some pair $\left(t_{i}, t_{j}\right)$ bounds $c p t$, then plan $p_{j}$ can be returned as 
the answer to getPlan. The complexity of this procedure is clearly quadratic in the size of $T_{Q}$. To avoid the enumeration of all of pairs of triples that have to be checked, we apply an optimization that allows us to choose a single pair of triples $\left(t_{1}, t_{2}\right)$ to be checked.

\section{Definition ( 4 (below) and $\gg$ (above) operators).}

Given a list, $T_{Q}$, of $k$ triples $\left(c p t_{i}, p_{i}\right.$, cost $\left._{i}\right)$ ordered by cost $t_{i}$, with $i=0, \ldots, k-1$, where cpt $t_{i}$ is a CostPoint, and cost $t_{i}$ represents the cost of executing the optimal plan $p_{i}$ at $c p t_{i}$, and given cpt, another CostPoint, we define the following operations:

1. $T_{Q} \varangle c p t$ is the list of triples $\left(c p t_{i}, p_{i}\right.$, cost $\left._{i}\right)$ from $T_{Q}$

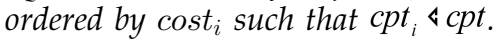

2. $T_{Q} \gg c p t$ is the list of triples $\left(c p t_{i}, p_{i}\right.$, cost $\left._{i}\right)$ from $T_{Q}$ ordered by cost $t_{i}$ such that cpt $_{i} \gg$ cpt.

Example 3. Let $T_{Q}=\left(t_{1}, t_{2}, t_{3}, t_{4}, t_{5}, t_{6}, t_{7}\right)$ be triples shown in a two-dimensional cost-based parameter space in Fig. 11. Then, $T_{Q} \varangle c p t=\left(t_{1}, t_{3}\right)$ (the triples in the light gray area), and $T_{Q}>c p t=\left(t_{5}, t_{6}, t_{7}\right)$ (the triples in the dark gray area).

As shown in Example 2 in the previous section, there is potentially more than one solution to $\operatorname{getPlan}(Q, c p t)$. We next show that if there is a solution, we only need to check if cost $_{\text {last }} \leq \operatorname{cost}_{\text {first }} \leq \operatorname{cost}_{\text {last }} * M+A$, where $c_{\text {first }}$ is the cost of the first triple in $T_{Q}>c p t$, and $c_{\text {last }}$ is the cost of the last triple in $T_{Q} \varangle c p t$. Then, in such situation, the plan in the first triple of $T_{Q}>c p t, p_{\text {first, }}$ is returned. Theorem 2 proves the correctness of this approach.

Theorem 2. If $\exists c p t_{b}: t_{b}=\left(c p t_{b}, p_{b}\right.$, cost $\left._{b}\right), \quad t_{b} \in T_{Q} \varangle c p t$, $\exists c p t_{a}: t_{a}=\left(c p t_{a}, p_{a}, \operatorname{cost}_{a}\right), t_{a} \in T_{Q}>c p t$, and $\operatorname{cost}_{b} \leq \operatorname{cost}_{a} \leq$ $\operatorname{cost}_{b}{ }^{*} M+A$, then cost $_{\text {last }} \leq$ cost $_{\text {first }} \leq \operatorname{cost}_{\text {last }} * M+A$, where cost $_{\text {first }}$ is the cost of the first triple in $T_{Q}>c p t$, and cost $_{\text {last }}$ is the cost of the last triple in $T_{Q}$ scpt.

Proof. By definition, the CostPoint of any triple that belongs to the below list is below the CostPoint of any triple that belongs to the above list. Formally,

$$
\forall c p t_{b}: t_{b}=\left(c p t_{b}, p_{b} \cos t_{b}\right) \in T_{Q} \varangle c p t
$$

and $\forall c p t_{a}: t_{a}=\left(c p t_{a}, p_{a}, \cos t_{b a}\right) \in T_{Q}>c p t$, we have that $c p t_{b} \triangleleft c p t \varangle c p t_{a}$. Then, by Lemma 1 , we have that $\operatorname{cost}_{b} \leq \operatorname{cost}_{\text {last }} \leq \mathrm{Opt}(\mathrm{cpt}) \leq \operatorname{cost}_{\text {first }} \leq \operatorname{cost}_{a}$. By $\operatorname{cost}_{b} \leq$ $\operatorname{cost}_{a} \leq \operatorname{cost}_{b} * M+A$ and Lemma 2, it follows that $\operatorname{cost}_{\text {last }} \leq \operatorname{cost}_{a} \leq \operatorname{cost}_{\text {last }}{ }^{*} M+A$. Also, if $\operatorname{cost}_{x} \leq$ $\operatorname{cost}_{z} \leq \operatorname{cost}_{x}{ }^{*} M+A$ and $\operatorname{cost}_{x} \leq \operatorname{cost}_{y} \leq \operatorname{cost}_{z}$, then $\operatorname{cost}_{x} \leq \operatorname{cost}_{y} \leq \operatorname{cost}_{x}{ }^{*} M+A$. Putting all together, it follows that cost $_{\text {last }} \leq \operatorname{cost}_{\text {first }} \leq \operatorname{cost}_{\text {last }} * M+A$.

The optimized implementation of getPlan is shown in Fig. 12. We can see that given the properties of $T_{Q} \varangle c p t$ and $T_{Q} \gg c p t$, it is possible to select a single triple $t_{1}$ from $T_{Q} \varangle c p t$ and a single triple $t_{2}$ from $T_{Q}>c p t$ such that only pair $\left(t_{1}, t_{2}\right)$ needs to be checked. Note that the implementation of getPlan in Fig. 12 makes at most a single pass over $T_{Q}$; thus, it has $\mathrm{O}\left(\left|T_{Q}\right|\right)$ time complexity, where $\left|T_{Q}\right|$ is the number of elements in $T_{Q}$. (Note that the search condition depends on multiple attribute values - the cost parameters-and therefore, more sophisticated search procedures such as binary search are not applicable.) Before addPlan is

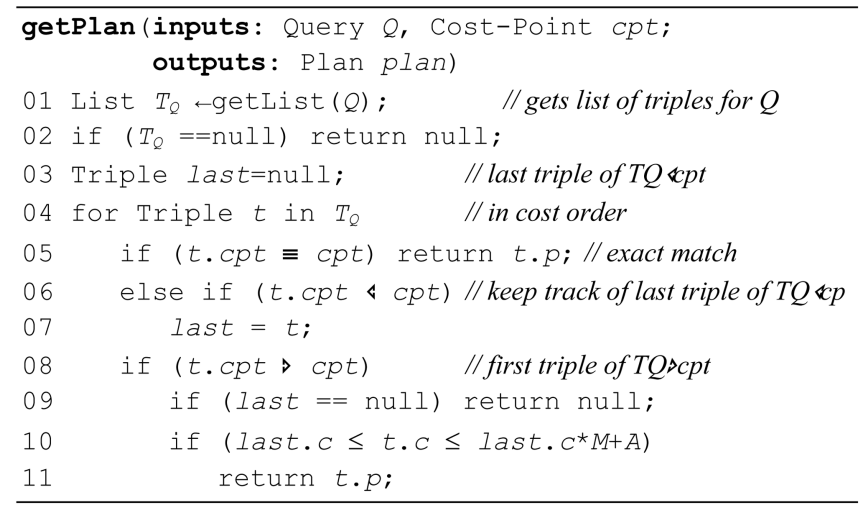

Fig. 12. Bounded's getPlan implementation.

called for the first time, any getPlan call returns null. As new triples are added, the hit rate of getPlan is expected to increase. Intuitively, as more triples are added, the more likely it is that getPlan returns a plan because it is more likely that any two triples fulfill the requirements of Theorem 2. Note also that the lower the values of $M$ and $A$, the less likely it is to find pairs of triples that fulfill the requirements of Theorem 2, and thus, more added triples are needed to obtain higher hit rates.

\section{The ElLIPSE-PPQO IMPLEMENTATION}

Bounded's getPlan provides strong guarantees on the cost of plans returned. However, we expect low hit rates of Bounded's getPlan for small values of $M$ and $A$ or before Bounded's $T_{Q}$ has been populated. In this section, we propose the Ellipse-PPQO (or simply Ellipse) implementation of the PP interface, designed to address Goal 1 in Section 2.2 (i.e., having high hit rates). For that purpose, Ellipse's getPlan returns $\Delta$-acceptable plans rather than guaranteed near-optimal costs.

Definition ( $\Delta$-acceptable plans). For $\Delta \in[0,1]$, if plan $p$ is known to be optimal at points $c_{1} t_{1}$ and cpt $_{2}$ in the cost-based parameter space, then plan $p$ is $\Delta$-acceptable at point cpt in the cost-based parameter space if and only if

$$
\frac{\left\|c p t_{1}-c p t_{2}\right\|}{\left\|c p t-c p t_{1}\right\|+\left\|c p t-c p t_{2}\right\|} \geq \Delta,
$$

where $\|p-q\|$ is the euclidean distance between $p$ and $q$.

It follows from the definition of $\Delta$-acceptable that if $p$ is optimal at $c p t_{1}$ and $c p t_{2}$, then $p$ is 1-acceptable only on points between $c p t_{1}$ and $c p t_{2}$ and $p$ is 0 -acceptable at all points. Note that in a two-dimensional space, the area where $p$ is $\Delta$-acceptable is equivalent to the definition of an ellipse; if $p$ is optimal for $c p t_{1}$ and $c p t_{2}$, then $p$ is $\Delta$-acceptable at $c p t$ if $c p t$ is on or inside an ellipse of foci $c p t_{1}$ and $c p t_{2}$ such that the distance between the foci, $\left\|c p t_{1}-c p t_{2}\right\|$, over the sum of the distances between $c p t$ and the foci, $\left\|c p t-c p t_{1}\right\|+$ $\left\|c p t-c p t_{2}\right\|$, is at least $\Delta$. Fig. 13 shows the areas where $p$ is 0.5 -acceptable, 0.8 -acceptable, and 1-acceptable if $p$ is optimal at $c p t_{1}$ and $c p t_{2}$.

Ellipse-PPQO encodes the heuristic that if a plan $p$ is optimal in two points $c p t_{1}$ and $c p t_{2}$, then $p$ is likely to be optimal or near-optimal in a convex region that encloses $c p t_{1}$ and $c p t_{2}$. Note that a nearest neighbor algorithm could 


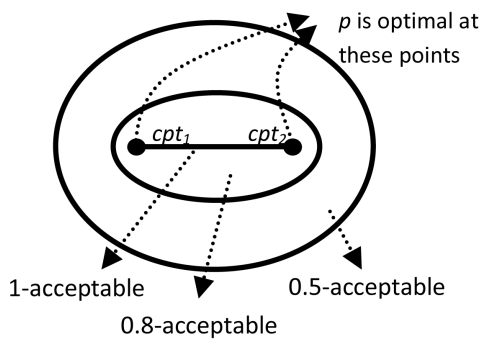

Fig. 13. Areas where $p$ is $\Delta$-acceptable.

\begin{tabular}{|c|c|}
\hline adc & $\begin{aligned} \text { Plan(inputs: } & \text { Query } Q, \text { CostPoint cpt, } \\
& \text { Plan } p, \text { Cost cost) }\end{aligned}$ \\
\hline 01 & PointList $L \leftarrow$ getPointList $(Q, p)$;// where is p optimal? \\
\hline 02 & if $(L==$ null) $/ /$ if no PointsList \\
\hline 03 & $L=$ new PointList ()$; / /$ create new one \\
\hline 04 & PlanList $P \leftarrow$ getPlanList $(Q)$; // optimal plans for $Q$ \\
\hline 05 & if $(P==$ null) $P=$ new PlanList $(p)$; \\
\hline 06 & else $\quad P$.insert $(p) ; / /$ add new optimal plan to list \\
\hline 07 & setPlanList $(Q, P) ; \quad / /$ adds/replaces list $P$ in catalog \\
\hline & L.insert (cpt, cost); \\
\hline & // adds/replaces list $\mathrm{L}$ in catalog \\
\hline
\end{tabular}

Fig. 14. Ellipse's addPlan implementation.

be used as an alternative to Ellipse-PPQO. However, since regions of optimality are frequently long and narrow [16], for any given cpt point, the closest known plan could very well be from another region of optimality (which we verified in practice). In addition, $\Delta$-acceptable areas can easily encode both small and large regions of optimality.

\subsection{Implementation of addPlan for Ellipse}

The implementation of addPlan for Ellipse proceeds as follows: For each query $Q$ and for each plan $p$ that is optimal in some point of the parameter space, Ellipse's addPlan $(Q, c p t, p, c o s t)$ essentially maintains a list of (cpt, cost) pairs, where $p$ is optimal for $Q$ (see Fig. 14).

\subsection{Implementation of getPlan for Ellipse}

Ellipse's getPlan (see Fig. 15) consists in the following. For each optimal plan $p$, it iterates over pairs of points where $p$ is optimal for the given query, $Q$. For each pair of points $\left(c p t_{1}, c p t_{2}\right)$, it tests if $p$ is $\Delta$-acceptable at the given point $c p t$. If it is, getPlan returns $p$; otherwise, getPlan keeps trying other points and plans. If all pairs of plans for $Q$ are exhausted without a $\Delta$-acceptable plan being found, getPlan returns null. Note that we return the first $\Delta$-acceptable plan, and therefore, getPlan depends on the order on which points are enumerated. Instead of returning the first match, we can consider all $\Delta$-acceptable plans and return the one with the largest distance from $\Delta$, which might improve the quality of the resulting plans at the cost of a slower implementation of getPlan.

\section{Experimental Evaluation}

In this section, we report an experimental evaluation of PPQO using Microsoft SQL Server 2005. The client application implements the pseudocode described in Sections 3 and 4, and Microsoft SQL Server is used to obtain estimated optimal plans and estimated costs of plans.

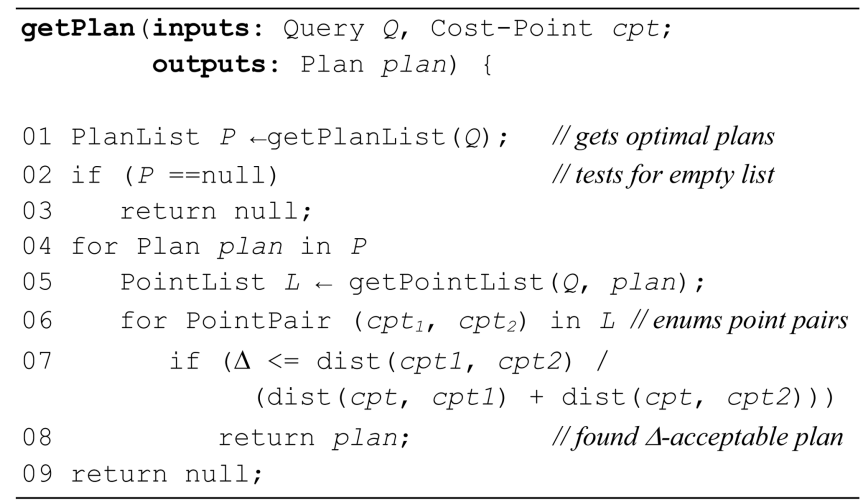

Fig. 15. Ellipse's getPlan implementation.

TABLE 1

Description of TPC-H Queries Used

\begin{tabular}{cccc}
\hline Query & Tables Joined & Column 1 & Column 2 \\
\hline 7 & LOCSNN & c_acctbal & o_totalprice \\
8 & LOCPSNNR & s_acctbal & 1_extendedprice \\
9 & LOTPSN & s_acctbal & 1_extended price \\
18 & LLOC & c_acctbal & 1_extendedprice \\
& & & \\
21 & LLLOSN & s_acctbal & 1_extendedprice \\
\hline
\end{tabular}

\subsection{Data Set, Metrics, and Setup}

The TPC-H benchmark [17] was used to evaluate the PPQO implementations. Table 1 shows which tables are joined by each query. The tables are lineitem $(\mathrm{L})$, orders $(\mathrm{O})$, customer $(\mathrm{C})$, supplier $(\mathrm{S})$, part $(\mathrm{P})$, partsupp $(\mathrm{T})$, nation $(\mathrm{N})$, and region $(\mathrm{R})$.

As in the work of Reddy and Haritsa [16] and unless otherwise noted, we added two extra selections to the TPC-H queries to more easily explore the parameter space (see Section 5.7 for experiments with more than two selection predicates). The two selections are of the form $\mathrm{col}_{i} \leq \mathrm{val}_{i}, i=1,2$, where for each query, $\mathrm{col}_{i}$ is one of the two columns shown in Table 1 , and $v^{2} l_{i}$ is a random value from the domain of the column.

For each query tested, we generated 10,000 random $v l_{1}$ and $v_{a l}$ values. (A $\left(v_{1} l_{1}, v a l_{2}\right)$ pair is a ValuePoint.) To guarantee that random parameter values uniformly explore the parameter space, we altered the values in the columns subject to the extra selections to such that those values are uniformly distributed in their domains instead of using the nonuniform TPC-H generated distributions.

For each query and each ValuePoint vpt, we make a getPlan lookup call (see Fig. 5), where PP is either OptimizeOnce, Optimize-Always, Bounded, or Ellipse. If getPlan returns a plan, we call it a hit and check if the plan is optimal; if it is not optimal, we check how its estimated cost compares with the estimated optimal cost. These give rise to the following metrics:

- HitRate. This metric refers to the fraction of getPlan calls that return a plan.

- OptRate. This metric refers to the percentage of plans that are optimal.

- SO. This metric refers to the measure of suboptimality: $p_{h i t}(c p t) / O p t(c p t)$, with $p_{h i t}=\operatorname{getPlan}(Q, c p t)$. $S O \geq 1$. 


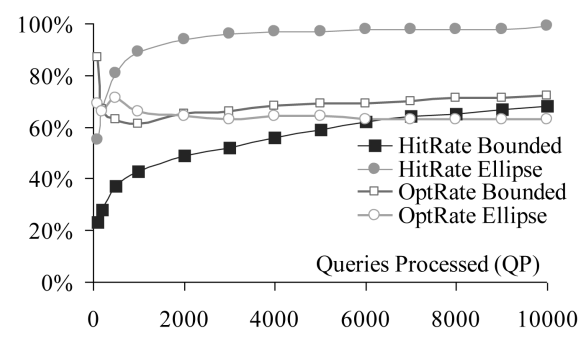

Fig. 16. HitRate and OptRate for Query 7.

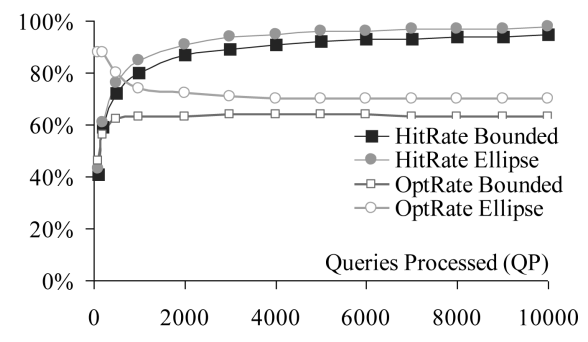

Fig. 17. HitRate and OptRate for Query 8.

- AvgSO. This metric refers to the average of all SO values.

- MaxSO. This metric refers to the maximum of all SO values and reflects how risky a PP implementation can be.

- Number of points. This metric refers to the number of $(c p t, p l a n, c o s t)$ triples stored in a ParametricPlan (i.e., number of misses).

- Number of plans. This metric refers to the number of distinct optimal plans.

- QP. This metric refers to the number of queries processed.

The experiments were run on a lightly loaded Pentium $\mathrm{M}$ at $1.73 \mathrm{GHz}$ with 1 Gbyte of RAM and using TPC-H scale factor 1 . Indexes and statistics were built on all columns subject to selections and on all primary and foreign key columns. To estimate the cost of suboptimal plans returned by PPQO, each suboptimal plan was forcibly cost by SQL Server [13]. Unless otherwise stated, Bounded used $M=1.1$ and $A=0$, and Ellipse used $\Delta=0.95$.

\subsection{Variation on HitRate and OptRate}

The first experiment consisted of processing queries using 10,000 different random ValuePoints (value vectors) for each query and observing how HitRate and OptRate varied for Bounded and Ellipse. This experiment was performed for the five TPC-H queries listed in Table 1, and the results for three are shown in Figs. 16, 17, and 18. Several trends can be observed:

- Ellipse always has a higher HitRate than Bounded.

- Except for Query 8 (more on this below), Bounded always has a higher OptRate than Ellipse.

- HitRate converges quickly, but OptRate converges slightly faster.

- HitRate monotonically increases as a function of QP (more processed queries imply more misses, and each miss adds information to the ParametricPlan, therefore increasing the likelihood of future hits).

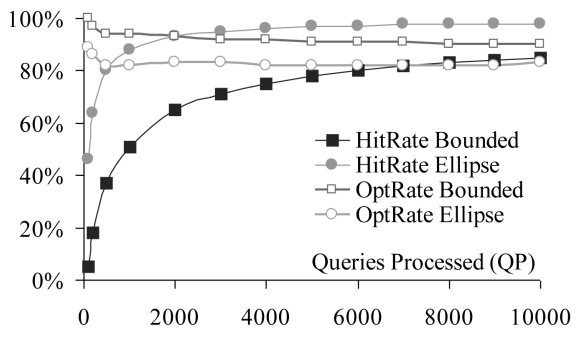

Fig. 18. HitRate and OptRate for Query 21.

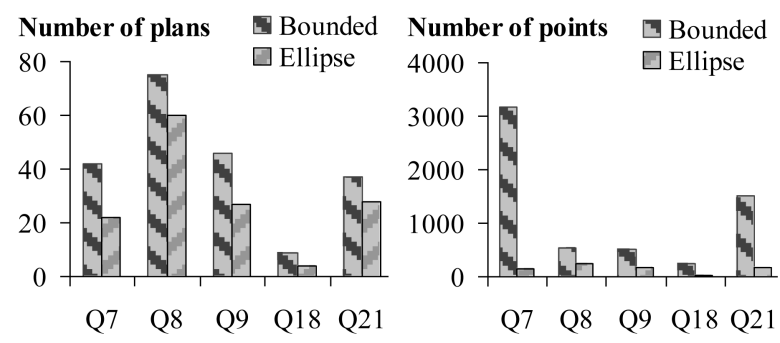

Fig. 19. Number of plans and points for 10,000 QP

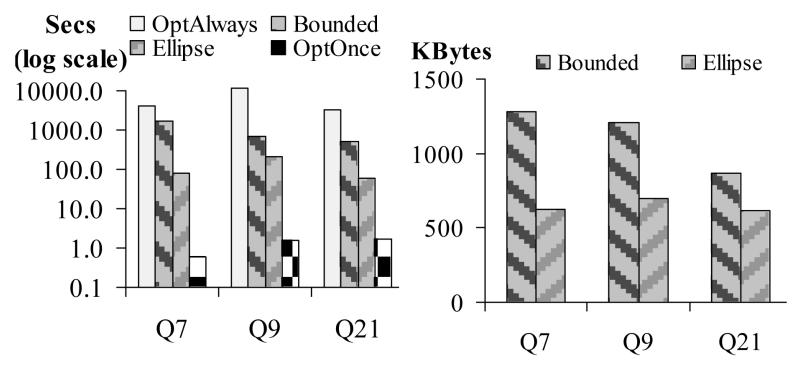

Fig. 20. Optimization time and space for 10,000 QP.

- OptRate naturally varies up and down, as the initial random (cpt,plan, cost) triples are added to the ParametricPlan object, until it converges.

\subsection{Number of Plans and of Points, Space, and Time}

Fig. 19 shows the number of plans and the number of points for the experiments of the previous section. Bounded has a higher number of plans and number of points because it has a lower HitRate; for every miss, there will be a new point stored in the ParametricPlan object.

Storing the number of plans and the number of points took only between $\sim 600$ Kbytes to $\sim 1,300$ Kbytes using the original uncompressed XML plan representations provided by SQL Server. Storing zip-compressed XML plans instead would decrease the size of the plan representation by a factor of 10. (Plans do not need to be understood, zipped, or unzipped by addPlan or getPlan functions.)

Fig. 20 reports the time and space taken by the Bounded and Ellipse approaches during optimization. Time (in seconds) includes the time elapsed during optimization (if there is a miss), during addPlan, and during getPlan but not the execution time nor the time consumed by function $\varphi$. For comparison purposes, the time taken for Optimize-Once and Optimize-Always is also included.

After 10,000 queries have been processed, OptimizeAlways took between 5.2 and 13.6 times longer than Bounded and between 10.7 and 18.5 times longer than Ellipse. Thus, although Bounded only used between 7 percent and 20 percent of the optimization time, it still returned plans 


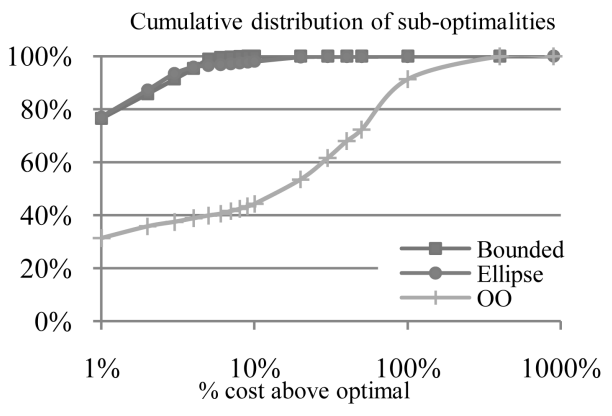

Fig. 21. Quality of returned plans (Q7).

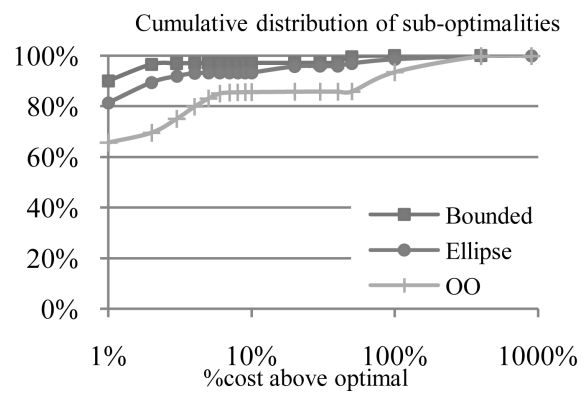

Fig. 22. Quality of returned plans (Q9).

that were, as shown in Section 5.4, on the average just 1 percent more costly than the optimal plan. Ellipse used between 5 percent and 9 percent of the optimization time and returned plans that were 6 percent more costly than the optimal plan. Ellipse was always faster than Bounded because it had less optimize and addPlan calls (due to higher HitRates) and faster getPlan calls (because it has less information stored in its PPs).

Note that although Optimize-Once spends the least optimization time, it is not the best overall approach (as seen in Fig. 24). In fact, the entire PQO research area aims to overcome the performance problems of using Optimize-Once.

\subsection{Quality of Returned Plans}

The quality of the returned plans is described in this section. The suboptimality of each plan returned by Bounded, Ellipse, and Optimize-Once was measured in the same experiments of the previous two sections.

Figs. 21, 22, and 23 show the quality of the returned plans (hits) for Bounded, Ellipse, and Optimize-Once in the form of cumulative distributions. The $x$-axis represents how much the cost of a returned plan is above optimal, and the $y$-axis represents the cumulative percentage of plans that correspond to that suboptimality level. For example, about 77 percent of the plans returned by Bounded for Query 7 are within 1 percent of the cost of optimal, and 99.9 percent are within 10 percent the cost of optimal. The quality of most plans returned by Ellipse and Bounded is very good, and the quality of the plans returned by Bounded is higher.

To complete the picture, Fig. 24 shows the average and maximum suboptimality for the three policies and five queries. While both Bounded and Ellipse have very good average cases, Ellipse can have as bad worse cases as Optimize-Once (but less frequently). Overall, Bounded's most suboptimal plan was five times worse than the optimal plan, while the most suboptimal plan chosen by

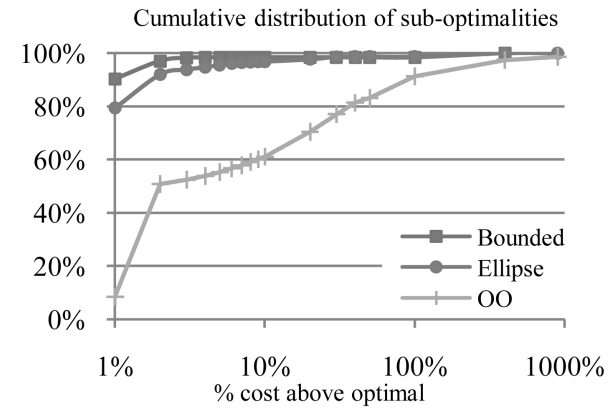

Fig. 23. Quality of returned plans (Q21).

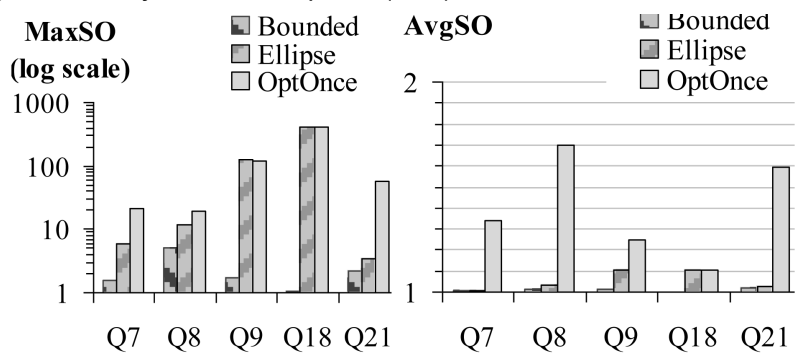

Fig. 24. MaxSO and AvgSO.

both Ellipse and Optimize-Once was 412 times more costly than the optimal plan (MaxSO graph in Fig. 24).

An interesting observation is that although Bounded (with $\mathrm{M}=1.1$ ) is supposedly guaranteed to return plans no more than 110 percent the cost of the optimal plan, in some experiments, that guarantee was violated. Indeed, for queries $7,8,9$, and 21 , the most suboptimal plan returned by Bounded was, respectively, 155 percent, 499 percent, 172 percent, and 177 percent the cost of the corresponding optimal plan. Further analysis showed that the problem lied with the tool that forces plans and that obtains the estimated cost of those plans. In some very rare cases, for a specific CostPoint cpt, the tool returned a plan, say, $p_{1}$ with cost $c_{1}$ at $c p t$, as if it was optimal, but some other plan, say, $p_{2}$, had an estimated cost $c_{2}$ at $c p t$ lower than $c_{1}$. This led to two problems: 1) Bounded stored plans and costs in its data structures that were not optimal, and 2) the costs of the (presumed) optimal plan appeared nonmonotonic. Other than those very rare occasions, Bounded guaranteed its suboptimality specifications. (Arguably, this issue affected Ellipse less because the Ellipse implementation does not rely on monotonic cost functions.)

Another surprise was how well Optimize-Once did in the AvgSO metric. On the average, across all queries, Optimize-Once returned plans with costs $\sim 140$ percent the cost of optimal (the same average was 101 percent for Bounded and $\sim 106$ percent for Ellipse). One possible explanation is the following. Optimize-Once obtains the optimal plan for the first of the 10,000 random parameter values and reuses that plan for all other values. If that first plan is also the plan with less cost variation in the plan space, then there is a high chance that that plan will do well in many other points in the space. Consider Fig. 25, which shows a conceptual representation of the costs of four different plans, each optimal in different regions of the parametric space.

Executing either plan $\mathrm{p}_{3}$ or plan $\mathrm{p}_{4}$ for all points of the parameter space would yield costs, on the average, not 


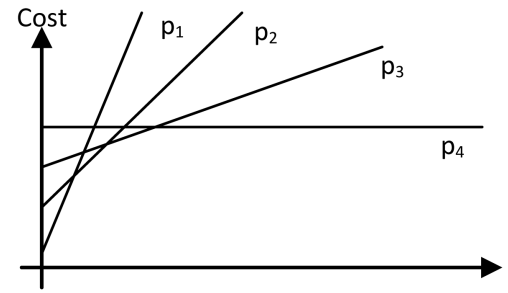

Fig. 25. Typical costs of optimal plans.

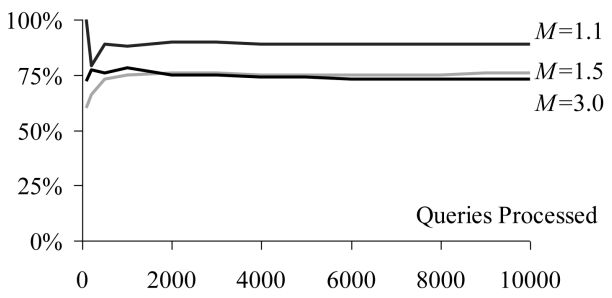

Fig. 26. OptRate for Bounded, vary M, Q21.

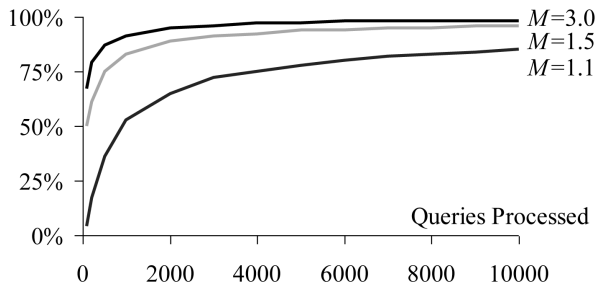

Fig. 27. HitRate for Bounded, vary M, Q21.

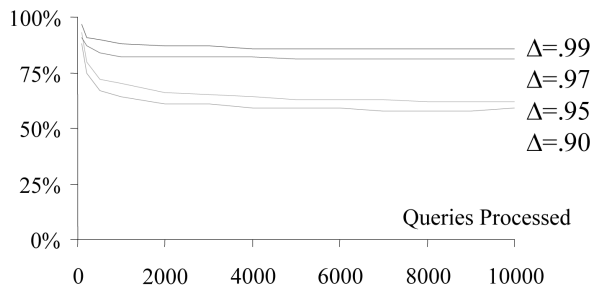

Fig. 28. OptRate for Ellipse, vary $\Delta, \mathrm{Q} 21$.

much higher than the cost of the optimal. Coincidentally, the likelihood that any given point lies in the space where either $\mathrm{p}_{3}$ or $\mathrm{p}_{4}$ are optimal is very high, and thus, by random chance, Optimize-Once is likely to use a plan that is not catastrophic. However, Optimize-Once can and will return catastrophic plans eventually. We will explore this issue further in Section 5.6-Vary Query Order.

\subsection{Vary Bounded's $M$ and Ellipse's $\Delta$}

In this experiment, the value $M$ of Bounded was varied from 1.1 to 3 for Query 21. The values of OptRate and HitRate are shown in Figs. 26 and 27. As expected, a lower value for $M$ (tighter optimality bound) results in a higher OptRate (because returned plans cannot be much worse than the corresponding optimal plans due to the tight optimality bound $\mathrm{M}$ ) but a lower HitRate (because tight values of $M$ result in small regions with quality guarantees, and therefore, a larger number of calls do not return any plan). Because the HitRate for $\mathrm{M}=1.5$ is already so close to 100 percent (Fig. 27), increasing $\mathrm{M}$ to 3 barely improves HitRate or change OptRate much. Alternatively, it could have resulted in a small change in HitRate but a larger change in OptRate (as it does not happen, there might be a

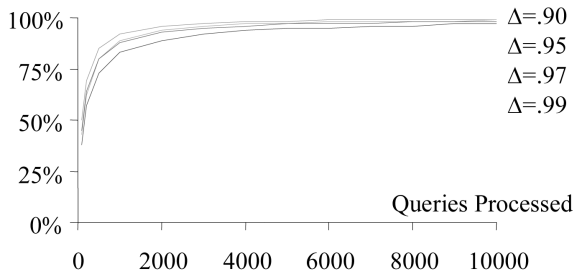

Fig. 29. HitRate for Ellipse, vary $\Delta$, Q21.

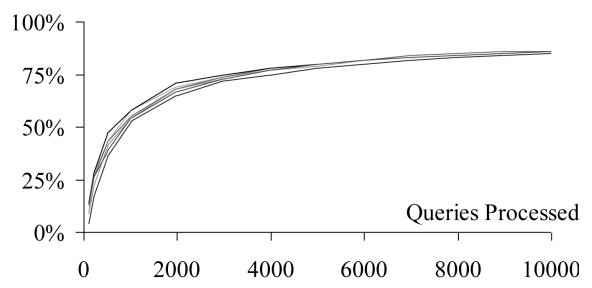

Fig. 30. HitRate for Bounded, vary query order, Q21.

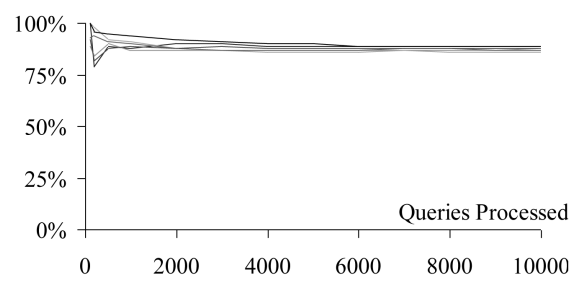

Fig. 31. OptRate for Bounded, vary query order, Q21.

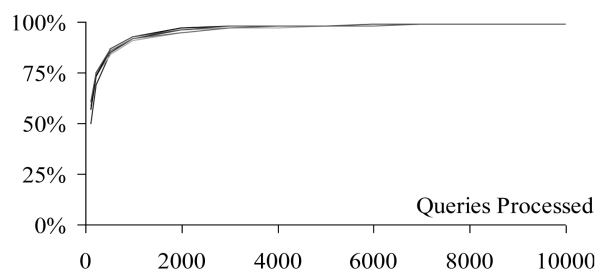

Fig. 32. HitRate for Ellipse, vary query order, Q21.

correlation between HitRate and OptRate in this scenario). The same Query 21 with the same random parameter values was run using Ellipse while varying $\Delta$ from 0.9 to 0.99 (see Figs. 28 and 29). As expected, a higher $\Delta$ results in a higher OptRate but a lower HitRate (the reasons are similar to those above). Due to space constraints, we do not report experiments varying Bounded's $A$ parameter. (The results, however, were similar to the ones for $\mathrm{M}$, i.e., larger values of $\mathrm{A}$ increase HitRate and decrease OptRate.)

\subsection{Vary Query Order}

This experiment assessed the impact of the order of the incoming queries on the performance of the algorithms. The same 10,000 random values used for Query 21 were used again, but the order in which those 10,000 queries were processed was chosen randomly. Six random orders were generated and processed with Bounded $(M=1.1, A=0)$, Ellipse $(\Delta=0.9)$, and Optimize-Once.

The results are shown in Figs. 30, 31, 32, and 33 and summarized in Table 2. Note that in Figs. 30, 31, 32, and 33, it is not possible to tell apart which line is which. That is precisely the point: except for Ellipse's OptRate, query order essentially had no effect on the values of HitRate or OptRate.

Note that although query order had no impact on the final values of Bounded's OptRate, Bounded's HitRate, and 


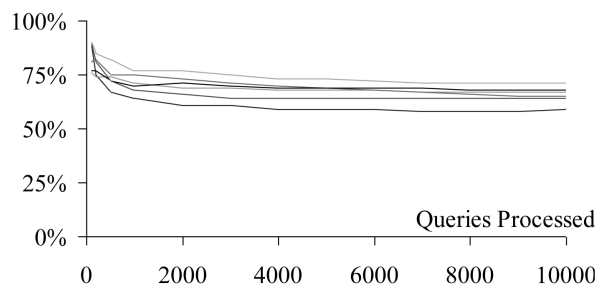

Fig. 33. OptRate for Ellipse, vary query order, Q21.

TABLE 2

Effects of Different Query Orders

\begin{tabular}{|r|ccc|ccc|}
\cline { 2 - 7 } \multicolumn{1}{c|}{} & \multicolumn{3}{c|}{ Final OptRate } & \multicolumn{3}{c|}{ Final HitRate } \\
& Max & Min & Avg & Max & Min & Avg \\
\hline Bounded & $89.0 \%$ & $86.0 \%$ & $87.8 \%$ & $86.0 \%$ & $85.0 \%$ & $85.8 \%$ \\
Ellipse & $71.0 \%$ & $59.0 \%$ & $65.7 \%$ & $99.0 \%$ & $99.0 \%$ & $99.0 \%$ \\
OptOnce & $48.0 \%$ & $3.0 \%$ & $35.2 \%$ & - & - & - \\
\hline
\end{tabular}

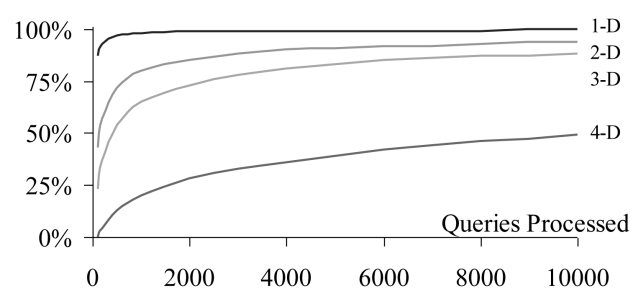

Fig. 34. Vary dimensions, HitRate for Bounded, Q8.

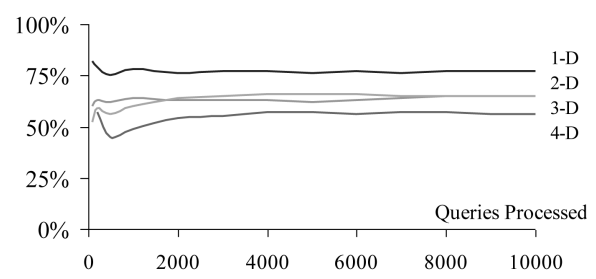

Fig. 35. Vary dimensions, OptRate for Bounded, Q8.

Ellipse's HitRate, query order did have a medium impact on the final value of Ellipse's OptRate.

On the other hand, for Optimize-Once, query order had a very significant impact on OptRate, with final values ranging from 3 percent to 48 percent. An interesting observation is that the performance of Optimize-Once was exactly the same for four out of those six random orders. Further analysis showed that although the very first value of each of the six random orders was different, for four of them, the corresponding optimal plan was the same. This follows the observation (Section 5.4, Fig. 25, and [16]) that some plans have very large optimality areas.

\subsection{Vary the Number of Dimensions}

In all the experiments so far, the parameter space was twodimensional. The next experiment varies the number of dimensions, from one to four. Query 8 is used (with extra parametric selections as needed) because it was the one with the highest number of plans and, thus, more likely to suffer from the "curse of dimensionality": an exponential growth of complexity with a linear increase in the number of dimensions. The query was then run for 10,000 random values for Bounded $(M=1.1, A=0)$ and Ellipse $(\Delta=0.95)$.

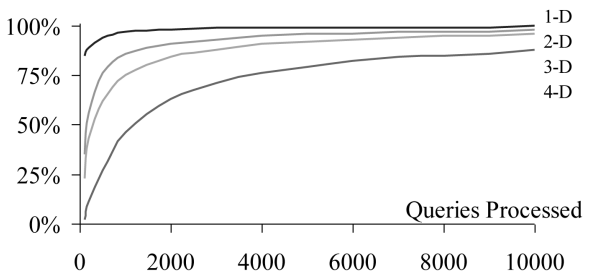

Fig. 36. Vary dimensions, HitRate for Ellipse, Q8.

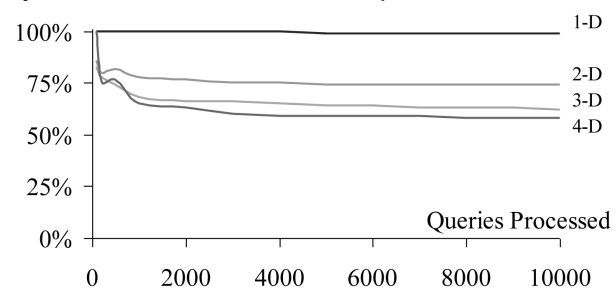

Fig. 37. Vary dimensions, OptRate for Ellipse, Q8.

TABLE 3

Variation of the Number of Dimensions

\begin{tabular}{|r|cccc|cccc|}
\cline { 2 - 8 } \multicolumn{1}{c|}{} & \multicolumn{4}{c|}{ OptRate } & \multicolumn{4}{c|}{ HitRate } \\
\cline { 2 - 9 } \multicolumn{1}{c|}{ 1-D } & 2-D & 3-D & 4-D & 1-D & 2-D & 3-D & 4-D \\
\hline Bounded & $77 \%$ & $65 \%$ & $65 \%$ & $56 \%$ & $100 \%$ & $94 \%$ & $88 \%$ & $49 \%$ \\
Ellipse & $99 \%$ & $74 \%$ & $62 \%$ & $58 \%$ & $100 \%$ & $98 \%$ & $96 \%$ & $88 \%$ \\
\hline
\end{tabular}
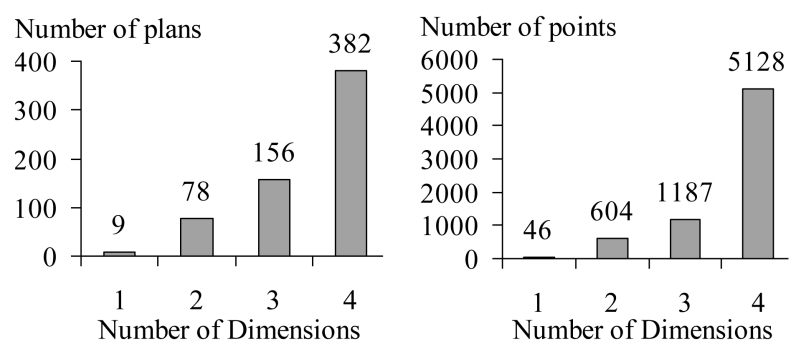

Fig. 38. Number of plans and points, Bounded, Q8.

The results, shown in Figs. 34, 35, 36, and 37, are summarized in Table 3.

It is clear that the more dimensions the parameter space has, the lower the OptRate and HitRate are. Some of the reasons that contribute to this effect are twofold. First, given a point $c p t$ centered in the middle of the parameter space, the percentage of space $4 c p t$ (or $>c p t$ ) decreases exponentially with the number of dimensions (affects Bounded). That is, the larger the number of dimensions, the less likely it is that any two random points are above or below some other point. For example, for a one-dimensional space, 50 percent of space is below (above) the midpoint. For two-dimensional, 25 percent of the parameter space is below (above) the midpoint (12.5 percent for three-dimensional and $\sim 6$ percent for four-dimensional). Fig. 38 shows the number of plans and points for Bounded. Second, the number of unique optimal plans increases exponentially with the dimensionality of the parameter space. This issue affects Ellipse because this approach relies on finding two close-by points where the same plan is optimal. Fig. 39 shows the number of plans and points for Ellipse.

The number of plans and points increase exponentially for both Ellipse and Bounded, but it is slower for Ellipse. 


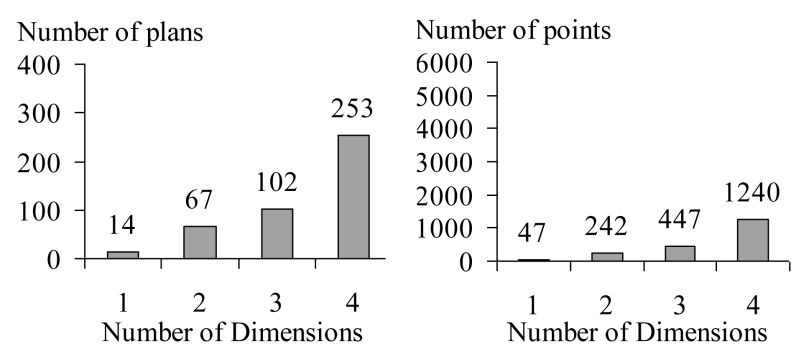

Fig. 39. Number of plans and points, Ellipse, Q8.

For each of the experiments above (which use one, two, three, and four cost parameters), the returned plans were on the average 7 percent, 8 percent, 45 percent, and 35 percent, respectively, more expensive than the optimal plans when using Ellipse and 0.2 percent, 2 percent, 24 percent, and 10 percent, respectively, more expensive than the optimal plans when using Bounded (not shown in the graphs).

\section{Related Work}

PQO was first mentioned by Graefe and Ward [7] and Lohman [12]. This pioneering early work also proposed dynamic query plans and a new metaoperator, the choose-plan [7]. Dynamic query plans include more than one physical plan choice. The plan to use is determined at runtime by the choose-plan operator after it costs the alternatives given the now known parameter values. How to enumerate dynamic query plans was proposed only later [2] with the concept of incomparability of costs: in the presence of unbound parameters at optimization time, plan costs are represented as intervals, and if intervals of alternative plans overlap, none is pruned. At runtime, when parameters are bound to values, the choose-plan selects the right plan. This approach may enumerate a large number of plans (see [15]), and all those plans may have to be recost at runtime. Ioannidis et al. [10] coined the term PQO and proposed using randomized algorithms to optimize in parallel the parametric query for all possible values of unknown variables. This approach is unfeasible for continuous parameters, gives no guarantees on finding the optimal plan for a query, and places no bounds on the optimality of the plans produced. Ganguly [5] uses a geometric approach to solve the PQO problem for one and two parameters under the assumption that cost functions are linear and that regions of optimality of plans are convex. Ganguly solves PQO for restricted forms of nonlinear one-parameter cost functions. Prasad [14] extends the geometric approach to solve PQO for ternary linear cost functions and binary nonlinear functions. Hulgeri and Sudarshan [8] propose a solution to $\mathrm{PQO}$ that handles piecewise linear cost functions for an arbitrarily number of parameters but requires substantial changes to the query optimizer. AniPQO [9] is a recent technique that approximates the solution to $\mathrm{PQO}$ for nonlinear functions and for an arbitrary number of parameters. AniPQO approximates optimality regions to $n$-dimensional polytopes and finds its solution to PQO by calling the optimizer multiple times and evaluating plan costs up to thousands of times. Unlike AniPQO, PPQO never calls the optimizer or costs plans more often than what a traditional non-PQO approach would.

A closely related piece of work is PLASTIC [6]. Like PPQO, PLASTIC incrementally maintains clusters of in- coming queries and avoids optimizing a new query if it is "close enough" to a previously seen cluster. At a high level, we can see PLASTIC as an instance of PPQO, where getPlan compares an incoming query against each of the previously saved ones and reuses an old query plan if it is "close enough" to the current query, and addPlan adds a plan as a new cluster representative. In contrast to Bounded and Ellipse, query similarity in PLASTIC is measured as a distance between feature vectors that describe the queries (such as the number of relations in the query, the number and type of predicates, and estimated sizes of tables and intermediate relations). For that reason, PLASTIC has the potential to detect similarities between queries with similar structure but touching different tables (like "SELECT R.a FROM R JOIN S" and "SELECT T.b FROM T JOIN U"). In our work, we do not attempt to reuse plans across different queries, so a direct implementation of PLASTIC would always compare instances of the same query with different parameters. As a consequence, the distance metric between queries would result in the sum of differences in the cost parameters, and PLASTIC would reduce to performing nearest neighbor searches on the parameter space with a threshold that determines when a new cluster should be created. As such, PLASTIC cannot give worst case quality guarantees on the resulting plans (as Bounded does) nor is able to model long and narrow optimality regions (as Ellipse does). It is, however, an interesting implementation of PPQO that might be useful in certain scenarios.

Finally, the recent work in [16] coins the term "plan diagram" to denote a pictorial enumeration of the execution plan choices of a query optimizer over the selectivity space. This work shows, using plan diagrams, that assumptions commonly held by PQO (plan convexity, plan uniqueness, and plan homogeneity) do not hold. These discoveries do not affect Bounded-PPQO, which provides optimality guarantees. On the other hand, Ellipse-PPQO results in higher hit rates but gives no optimality guarantees on returned plans and may produce poor results for large $\Delta$-acceptable regions. Very recently, in a follow-up to [16], the authors propose to reduce the plan diagram for a given query by "collapsing" plans whose costs are close enough to each other [3]. This work shares with ours the notion that in many cases, obtaining near-optimal plans is sufficient and might lead to dramatic reductions in the number of plans to consider without sacrificing the quality of the optimization process. A crucial difference with our work is that [3] proceeds a posteriori, after optimizing the input query for all possible parameters (specifically, over a fine grid that is laid out over the parameter space). In contrast, PPQO is to progressively build a PP data structure with no long startup costs.

\section{CONCLUSIONS}

Before PPQO, processing parameterized queries was an allor-nothing approach: either the optimizer explores all the parameter space and computes the full PQO solution (traditional PQO) or it relies on luck and uses the very first plan it gets for a query. PPQO is able to progressively construct information about the parametric space and approximate optimality regions, being able to bypass the optimizer up to 99 percent of the times, while still returning plans within 5 percent of the cost-optimal plan for 99 percent of the cases. Unlike PQO, PPQO does not perform extra optimizer calls or extra plan-cost evaluation calls. At execution time, PPQO selects which plan to execute 
by using only the input cost parameters without recosting plans. PPQO is an adaptive technique that works prior to execution (and assumes the optimizer to be correct-just like any other PQO approach). Query reoptimization [11] and other adaptive query processing (AQP) approaches [1], [4] work during optimization and execution and assume that the optimizer can make mistakes or that the system characteristics change significantly during the execution of a single query. Also, PPQO is an interquery adaptive approach, while AQP are frequently intraquery optimization approaches.

PPQO is also amenable to be implemented in a complex commercial database system as it requires no changes in the optimization or execution processes. In fact, our PPQO prototype ran outside the DBMS server. For technical reasons, we did not implement function $\varphi$ ourselves but instead used SQL Server's cost model to transform value into cost parameters. For that reason, we did not evaluate the impact of such function in our experimental evaluation. However, it is important to note that function $\varphi$ can be implemented by simply manipulating in memory histograms (i.e., 200-int arrays), which is a negligible fraction of optimization time and would not have resulted in any noticeable difference in our experimental evaluation.

PPQO was evaluated in a variety of settings, with queries joining up to eight tables, with multiple subqueries, up to four parameters, and in plan spaces with close to 400 different optimal plans. PPQO yielded good results in all scenarios except for the Bounded algorithm in complex queries using a four-dimensional parameter space. However, even in this challenging scenario, Ellipse on the average executed plans just 3 percent more costly than the optimal, while avoiding 87 percent of all optimization calls.

\section{REFERENCES}

[1] S. Babu and P. Bizarro, "Adaptive Query Processing in the Looking Glass," Proc. Second Biennial Conf. Innovative Data Systems Research (CIDR), 2005.

[2] R.L. Cole and G. Graefe, "Optimization of Dynamic Query Evaluation Plans," Proc. ACM SIGMOD, 1994.

[3] D. Harish, P. Darera, and J. Haritsa, "On the Production of Anorexic Plan Diagrams," Proc. 33rd Int'l Conf. Very Large Data Bases (VLDB), 2007.

[4] A. Deshpande, Z. Ives, and V. Raman, "Adaptive Query Processing," Foundations and Trends in Databases, vol. 1, no. 1, pp. 1-140, 2007.

[5] S. Ganguly, "Design and Analysis of Parametric Query Optimization Algorithms," Proc. 24th Int'l Conf. Very Large Data Bases (VLDB), 1998.

[6] A. Ghosh, J. Parikh, V.S. Sengar, and J.R. Haritsa, "Plan Selection Based on Query Clustering," Proc. 28th Int'l Conf. Very Large Data Bases (VLDB), 2002.

[7] G. Graefe and K. Ward, "Dynamic Query Evaluation Plans," Proc. ACM SIGMOD, 1989.

[8] A. Hulgeri and S. Sudarshan, "Parametric Query Optimization for Linear and Piecewise Linear Cost Functions," Proc. 28th Int'l Conf. Very Large Data Bases (VLDB), 2002.

[9] A. Hulgeri and S. Sudarshan, "AniPQO: Almost Non-Intrusive Parametric Query Optimization for Nonlinear Cost Functions," Proc. 28th Int'l Conf. Very Large Data Bases (VLDB), 2003.

[10] Y.E. Ioannidis, R.T. Ng, K. Shim, and T.K. Sellis, "Parametric Query Optimization," Proc. 18th Int'l Conf. Very Large Data Bases (VLDB), 1992.

[11] N. Kabra and D.J. DeWitt, "Efficient Mid-Query Re-Optimization of Sub-Optimal Query Execution Plans," Proc. ACM SIGMOD, 1998.
[12] G.M. Lohman, "Is Query Optimization a "Solved" Problem?" Proc. Workshop Database Query Optimization, Oregon Graduate Center Technical Report 89-005, 1989.

[13] Microsoft Corp., "Plan Forcing Scenario: Create a Plan Guide That Uses a USE PLAN Query Hint," SQL Server 2005 Books Online, 2005.

[14] V.G.V. Prasad, "Parametric Query Optimization: A Geometric Approach," MSc thesis, IIT, Kampur, 1999.

[15] S.V.U. Maheswara Rao, "Parametric Query Optimization: A Non-Geometric Approach," master's thesis, IIT, Kampur, 1999.

[16] N. Reddy and J.R. Haritsa, "Analyzing Plan Diagrams of Database Query Optimizers," Proc. 31st Int'l Conf. Very Large Data Bases (VLDB), 2005.

[17] Transaction Processing Performance Council, The TPC-H Benchmark, http://www.tpc.org/, accessed, Mar. 2006.

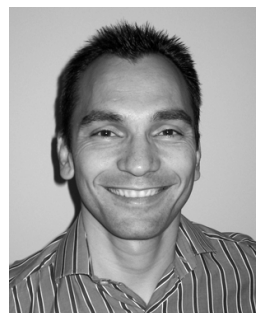

Pedro Bizarro received the $\mathrm{PhD}$ degree from the University of Wisconsin, Madison, in 2006. $\mathrm{He}$ is an assistant professor in the CISUC/DEI, University of Coimbra, Coimbra, Portugal. His research interests include adaptivity and query processing, stream systems, event processing systems, distributed systems, and benchmarking and performance. $\mathrm{He}$ is a Marie Curie fellow (under the European Union FP6 research grants), and he is leading the BiCEP-Bench marking Complex Event Processing Systems-research project.

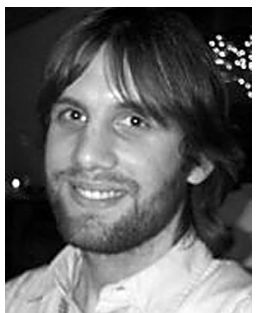

Nicolas Bruno received the BS degree in computer science from the School of Mathematics, Astronomy and Physics (FaMAF), Argentina, in 1998 and the PhD degree in computer science from Columbia University, New York, in 2003. He is currently a researcher in the Data Management, Exploration and Mining Group (DMX), Microsoft Research, Redmond, Washington. His research interests are in self-tuning database systems and query processing and optimization.

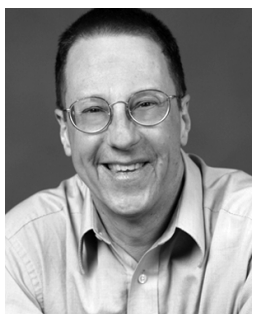

David J. DeWitt received the $\mathrm{PhD}$ degree from the University of Michigan in 1976. He joined the Computer Sciences Department, University of Wisconsin, Madison, in September 1976, where he served as the department chair from July 1999 to July 2004. He held the title John P. Morgridge Professor of Computer Sciences when he retired from the University of Wisconsin and joined Microsoft as a technical fellow in 2008. He received the 1995 SIGMOD Innovations Award for his contributions to the database systems field. He has authored more than 120 technical publications and served on numerous program committees and US National Science Foundation (NSF) Review Panels. He was a member of the NSF CISE Advisor Committee from 2000 to 2003 and the CSTB from 2005 to 2007 and has served on several NRC and DARPA study panels. He was the program chair of the 1983 SIGMOD Conference, a program cochair of the 1988 VLDB Conference, and the general chair of the 2002 SIGMOD Conference. He is a member of the National Academy of Engineering (1998), a fellow of the American Academy of Arts and Sciences (2007), and a fellow of the ACM (1995).

$\triangleright$ For more information on this or any other computing topic, please visit our Digital Library at www.computer.org/publications/dlib. 